Article

\title{
A Mathematical Model of Hourly Solar Radiation in Varying Weather Conditions for a Dynamic Simulation of the Solar Organic Rankine Cycle
}

\section{Taehong Sung ${ }^{1}$, Sang Youl Yoon ${ }^{2, *}$ and Kyung Chun Kim ${ }^{1, *}$}

1 School of Mechanical Engineering, Pusan National University, Busan 609-735, Korea;

E-Mail: taehongsung@pusan.ac.kr

2 Rolls-Royce and Pusan National University Technology Centre, Pusan National University, Busan 609-735, Korea

* Authors to whom correspondence should be addressed;

E-Mails: yoonsy@pusan.ac.kr (S.Y.Y.); kckim@pusan.ac.kr (K.C.K.);

Tel.: +82-51-510-2324 (K.C.K.); Fax: +82-51-515-7866 (K.C.K.).

Academic Editor: Mark Deinert

Received: 7 May 2015 / Accepted: 6 July 2015 / Published: 13 July 2015

\begin{abstract}
A mathematical model of hourly solar radiation with weather variability is proposed based on the simple sky model. The model uses a superposition of trigonometric functions with short and long periods. We investigate the effects of the model variables on the clearness $\left(k_{D}\right)$ and the probability of persistence $\left(P O P_{D}\right)$ indices and also evaluate the proposed model for all of the $k_{D}-P O P_{D}$ weather classes. A simple solar organic Rankine cycle (SORC) system with thermal storage is simulated using the actual weather conditions, and then, the results are compared with the simulation results using the proposed model and the simple sky model. The simulation results show that the proposed model provides more accurate system operation characteristics than the simple sky model.
\end{abstract}

Keywords: sky condition; hourly solar radiation; sky clearness index; probability of persistence; solar organic Rankine cycle; dynamic simulation 


\section{Introduction}

As a result of recent strong interest in solar energy, experimental solar radiation data for potential areas of solar power generation were collected and used in a solar energy system design. However, in many cases, those radiation data are insufficient. The operating characteristics and the performance of solar energy systems are unstable, since the amount of solar radiation gain and its pattern vary due to the changing of the solar collector efficiency or the thermal storage heat loss of a solar thermal energy system $[1,2]$. The data of solar radiation change in a short time are not cumulated for most potential solar energy system installation areas.

In general, solar systems use direct solar radiation observation, indirect solar radiation data, which are derived from observed temperature, humidity and other weather factors, or the averaged solar radiation data [3]. With observed solar radiation data, realistic system simulation and annual gain analysis are possible. Indirect solar energy estimate studies are conducted due to the lack of the actual solar radiation data [4-8]. Those estimations use observed weather variables, such as sunshine $[9,10]$, temperature [11,12] or cloud [11,13-15]. Using direct and indirect weather data involves significant effort in choosing data from the large original dataset. Therefore, this approach is problematic, since there are no criteria for sampling. The solar energy system is normally turned off when solar irradiation goes below a certain level (for example, $400 \mathrm{~W} / \mathrm{m}^{2}$ ), which means that the system is mainly operating during clear days. Freeman et al. [16] considered various ways of aggregating time-varying solar data and evaluated the energy output from a solar-thermal collector. They found that the extent of the error for the annual work output was caused by using averaged weather data.

The aim of this study is to add weather variability to a simple averaged solar radiation model; it will serve as a basis for the future development of an accurate solar radiation model for use in the design and development of new solar energy systems. To evaluate the proposed model, dynamic simulations of a solar organic Rankine cycle (SORC) system were conducted using an actual distribution of daily solar radiation, the proposed model and the simple sky model.

\section{Numerical Modelling of Solar Radiation}

The solar radiation model for a cloudy sky proposed in this study is based on the simple sky model [3] that is generally used in solar energy simulations. The simple sky model is characterized by the parameters of the peak solar irradiance $\left(S_{\max }\right)$, the sunrise time $\left(t_{\text {rise }}\right)$ and the sunset time $\left(t_{\text {set }}\right)$ as follows:

$$
I_{\text {simple sky }}=\left\{\begin{array}{c}
0, \mathrm{t} \leq t_{\text {rise }} \\
S_{\text {max }} \sin \left(\frac{\pi\left(t-t_{\text {rise }}\right)}{t_{\text {set }}-t_{\text {rise }}}\right), t_{\text {rise }}<t<t_{\text {set }} \\
0, \mathrm{t} \geq t_{\text {set }}
\end{array}\right.
$$

where $t$ is time. The peak solar irradiance is derived as equal total daily solar exposures between the observed value and the value from the simple sky model. The cloudiness $(M)$ is represented as the ratio of the peak solar irradiance $\left(S_{\max }\right)$ to the daily maximum extraterrestrial irradiance $\left(I_{E T I, \max }\right)$ as follows:

$$
S_{\max }=I_{E T I, \max } M
$$


The extraterrestrial irradiance $\left(I_{E T I}\right)$ is defined as the product of the solar constant ( $I_{0}$, fixed as 1362$)$, and the eccentricity correction factor $\left(E_{0}\right)$ that is usually represented using a simple correlation (see Equation (3)). Thus, the extraterrestrial irradiance is given by:

$$
I_{E T I}=I_{0} E_{0} \cos \theta_{z}=(1,362)\left(1+0.033 \cos \left(\frac{2 \pi d_{n}}{365}\right)\right) \cos \theta_{z}
$$

where $d_{n}$ is the number of the day in the year and $\theta_{z}$ is the zenith angle, which is calculated from the function of local time and location $[3,17]$.

As shown in Equation (1), the simple sky model consists of a single sinusoidal wave and therefore does not include the effects of weather variability.

To represent accurately the variation in the solar radiation due to weather changes, a superposition of multiple sinusoidal waves with different magnitudes and frequencies are considered. In this study, two sinusoidal waves are added to the simple sky model as follows:

$$
\begin{aligned}
I_{\text {cloudy sky }} & =S_{\text {max }} \sin \left(\frac{\pi\left(t-t_{\text {rise }}\right)}{t_{\text {set }}-t_{\text {rise }}}\right)\left(1-a_{1} *\left|\sin \left(b_{1} * \frac{\pi\left(t-t_{\text {rise }}\right)}{t_{\text {set }}-t_{\text {rise }}}\right)\right|^{c_{1}}\right. \\
& \left.-a_{2} *\left|\sin \left(b_{2} * \frac{\pi\left(t-t_{\text {rise }}\right)}{t_{\text {set }}-t_{\text {rise }}}\right)\right|^{c_{2}}\right), t_{\text {rise }}<t<t_{\text {set }}
\end{aligned}
$$

where $a_{1}$ and $a_{2}$ are the amount of solar irradiance variation; $b_{1}$ and $b_{2}$ are the frequency of the weather changes and $c_{1}$ and $c_{2}$ are the rate of change in weather.

Thus, the proposed model includes long (subscript 1) and short (subscript 2) periods of weather variations. In order to see how model variables effect solar irradiance distribution, a case study was conducted, and Figure 1 shows the distribution of solar radiation $I_{\text {cloudy,sky }}$ for the various values of the parameters $a_{1}, b_{1}, c_{1}$ and $b_{2}$. The effect of variable $\mathrm{a}_{1}$ is shown in Figure 1a. A higher value of a increases the depth of fluctuations. Figure $1 b$ shows the effect of the frequency of the long-term weather changes $b_{1}$. The number of $b_{1}$ indicates the number of fluctuations during a day. Figure $1 \mathrm{c}$ illustrates the effect of long-term weather change rate $c_{1}$. An increase in $c_{1}$ results in a clear increase in total daily solar irradiance. The effect of the short period weather changes $b_{2}$ is shown in Figure 1d. Similar to the case of $b_{1}, b_{2}$ represents the number of short-term fluctuations during a day.

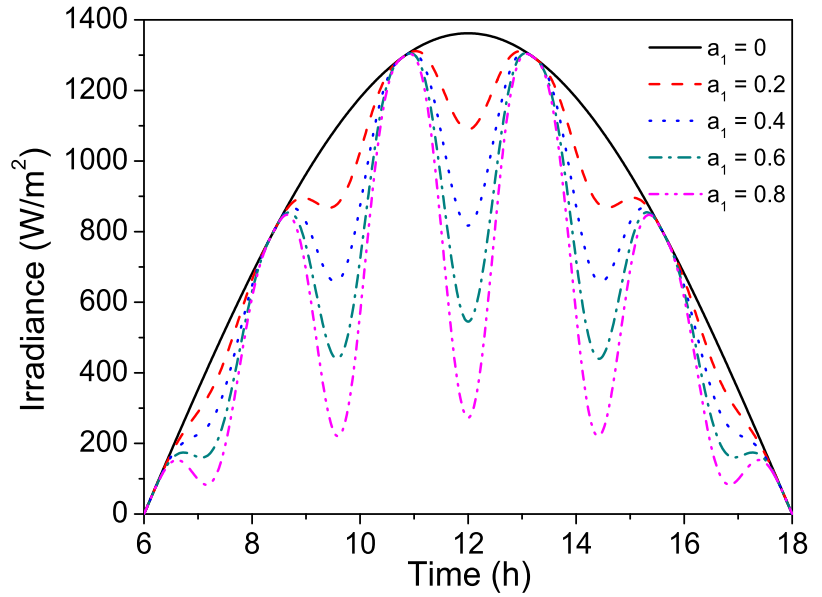

(a)

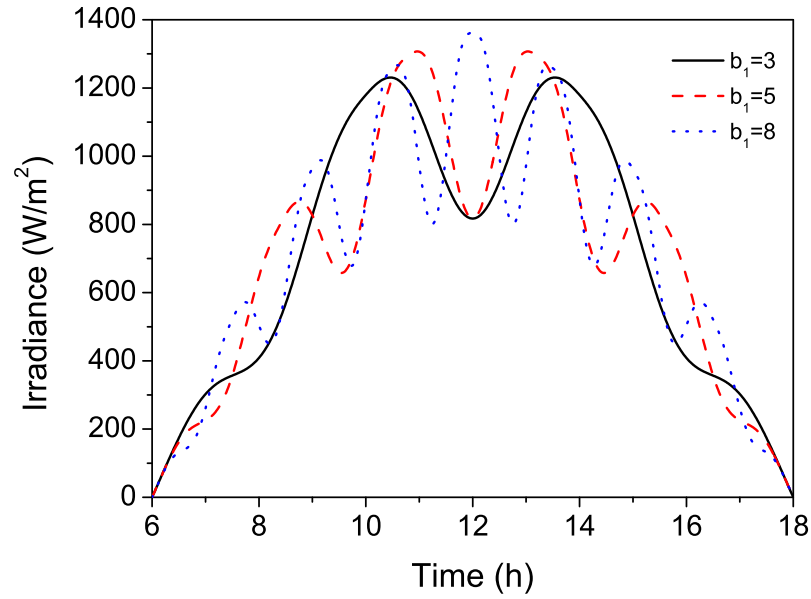

(b)

Figure 1. Cont. 


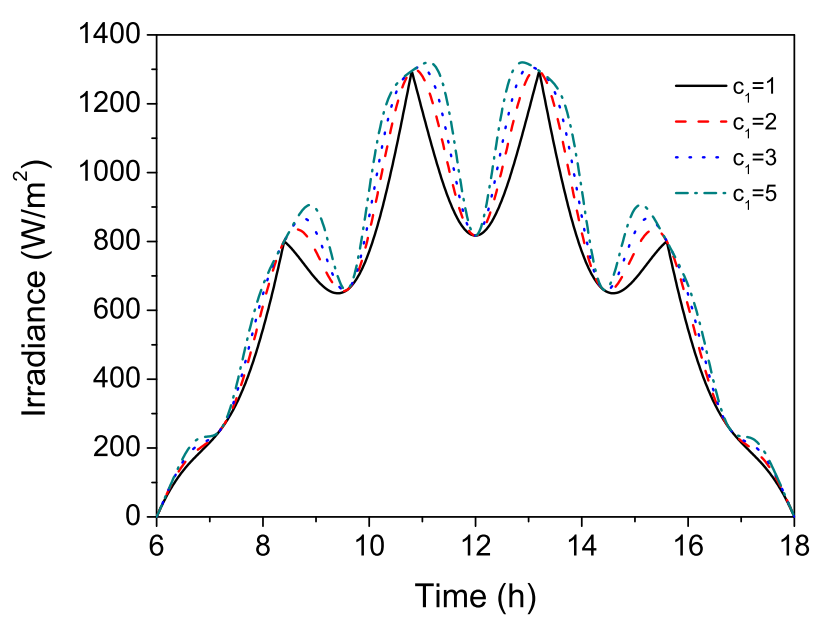

(c)

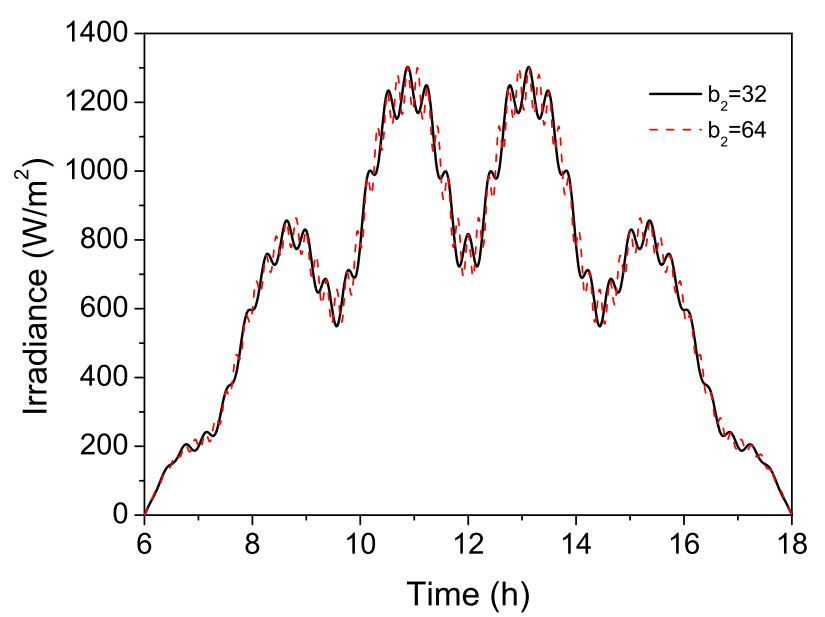

(d)

Figure 1. Solar radiation distributions obtained by the proposed model for the different values of the parameters in Equation (4): (a) $M=1, b_{1}=5, c_{1}=3, a_{2}=0$; (b) $M=1, a_{1}=0.3, c_{1}=3$, $a_{2}=0 ;$ (c) $M=1, a_{1}=0.3, b_{1}=5, a_{2}=0 ;$ (d) $M=1, a_{1}=0.3, b_{1}=5, c_{1}=3, a_{2}=0.1, c_{2}=3$.

Statistical indices are generally used for the characterization of solar radiation. The sky clearness index $\mathrm{k}$ is defined as the ratio of the actual received irradiance (instant global horizontal irradiance, $I_{G H I}$ ) to the maximum theoretically possible extraterrestrial irradiance $\left(I_{E T I}\right)$ :

$$
k=\frac{I_{G H I}}{I_{E T I}}=\frac{I_{G H I}}{I_{0} E_{0} \cos \theta_{z}}
$$

The daily sky clearness index $\mathrm{k}_{D}$ is then derived from the integrals of the variables in Equation (5) [3].

$$
k_{D}=\frac{\int_{t_{\text {rise }}}^{t_{\text {set }}}\left(I_{G H I}\right) d t}{\int_{t_{\text {rise }}}^{t_{\text {set }}}\left(I_{\text {ETI }}\right) d t}
$$

Kang and Tam [18] proposed the daily probability of persistence $\left(P O P_{D}\right)$ index that describes the stability of the daily solar irradiance for a fixed one-minute time interval. $P O P_{D}$ calculates a possibility that adjacent instantaneous clearness indices are equal. $P O P_{D}$ is derived from the following procedure.

1. Calculate the instantaneous clearness index $k$ for each time series.

2. Round off the value of $k$ at the first decimal place (e.g., 0.1, 0.3, etc.).

3. Count the number of identical cases between adjacent time series.

4. Divide the counting number by the number of time series $\left(P O P_{D}\right)$.

The higher value of $P O P_{D}$ indicates smaller weather variability. They also suggested a classification method based on $k_{D}$ and $P O P_{D}$ to describe the daily weather variability and showed that the observed daily weather could be categorized into ten classes, as shown in Table 1. 
Table 1. Classification parameters of solar radiation with $k_{D}$ and $P O P_{D}[18]$.

\begin{tabular}{|c|c|c|c|}
\hline Class & $k_{D}$ & $\mathbf{P O P}_{\mathbf{D}}$ & Description \\
\hline 1 & $k_{D}>0.6$ & $P O P_{D}>0.9$ & $\begin{array}{l}\text { High quantity and high quality; } \\
\text { sunny and steady sky conditions for almost the entire day }\end{array}$ \\
\hline 2 & $0.3<k_{D}<0.6$ & $P O P_{D}>0.9$ & $\begin{array}{l}\text { Medium quantity and high quality; partly cloudy, } \\
\text { but sky conditions are relatively steady for most of the day }\end{array}$ \\
\hline 3 & $k_{D}<0.3$ & $P O P_{D}>0.9$ & $\begin{array}{l}\text { Low quantity and high quality; overcast, } \\
\text { but sky conditions are relatively steady for most of the day }\end{array}$ \\
\hline 4 & $k_{D}>0.6$ & $0.7<P O P_{D}<0.9$ & $\begin{array}{l}\text { High quantity and medium quality; sunny, } \\
\text { but the sky conditions vary for part of the day }\end{array}$ \\
\hline 5 & $0.3<k_{D}<0.6$ & $0.7<P O P_{D}<0.9$ & $\begin{array}{l}\text { Medium quantity and medium quality; } \\
\text { partly cloudy and the sky conditions vary for part of the day }\end{array}$ \\
\hline 6 & $k_{D}<0.3$ & $0.7<P O P_{D}<0.9$ & Low quantity and medium quality; cloudy and the sky conditions vary \\
\hline 7 & $k_{D}>0.6$ & $0.5<P O P_{D}<0.7$ & $\begin{array}{l}\text { High quantity and low quality; partly sunny with sky conditions } \\
\text { varying significantly for most of the day }\end{array}$ \\
\hline 8 & $0.3<k_{D}<0.6$ & $0.5<P O P_{D}<0.7$ & $\begin{array}{l}\text { Medium quantity and low quality; various degrees of cloudiness and } \\
\text { the sky conditions varying significantly for most of the day }\end{array}$ \\
\hline 9 & $k_{D}<0.3$ & $0.5<P O P_{D}<0.7$ & $\begin{array}{l}\text { Low quantity and low quality; various degrees of cloudiness, } \\
\text { but high levels of fluctuations for the entire day }\end{array}$ \\
\hline 10 & - & $P O P_{D}<0.5$ & Very low quality \\
\hline
\end{tabular}

In order to examine the feasibility of the proposed model, four cases of the observed hourly solar irradiance samples are simulated. The solar radiation data are given by the National Renewable Energy Laboratory (http://www.nrel.gov/midc/) [19,20]. Each simulation curve is modelled according to the amount of solar irradiance $\left(k_{D}\right)$ and the characteristics of long- and short-term fluctuations. First, model shape variables of $a_{1}, a_{2}, b_{1}, b_{2}, c_{1}$ and $c_{2}$ are selected to implement the fluctuation pattern, and then, the amount of solar irradiance is matched by varying the value of cloudiness $M$. Solar radiation simulation variables and indices are shown in Table 2, and the corresponding irradiance distributions are shown in Figure 2. Figure 2a shows the irradiance distribution of the observed data and the simple model for a sunny day. During the daytime, the simple model returns relatively higher value of irradiance than the observed data, which make the thermal system warm-up faster during simulation. Figure $2 b$ illustrates the irradiance distributions for a cloudy day. It is obvious that the proposed model does not match with fluctuations in various frequencies and intensities compared to the curves by the observed data exactly, but follows the tendency of the irradiance distribution. Meanwhile, similar to sunny day results, the simple model and the proposed model show larger irradiance during daytime. Figure $2 \mathrm{c}, \mathrm{d}$ shows that the irradiance distribution of cloudy days is overcast, which is common. In this case, the proposed model does not fit well (Figure 2c). These kinds of weather changes can be simply implemented by removing fluctuation terms during, before or after a certain time (Figure $2 \mathrm{~d}$ ). Figure 2e,f illustrates the irradiance distribution of long-term large weather change conditions with short-term small fluctuations. Major differences between the cases are in the long term, such as the amount of solar irradiance $\left(a_{1}\right)$ being 0.3 and 0.6 and the frequency of the weather changes being six and seven. In all cases, the simulated curves match with the observed one within a specific period of time. 


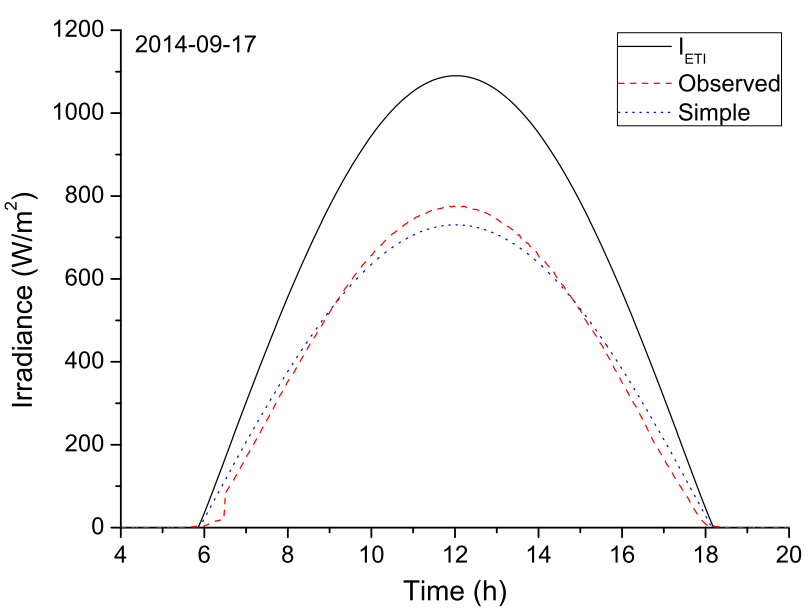

(a)

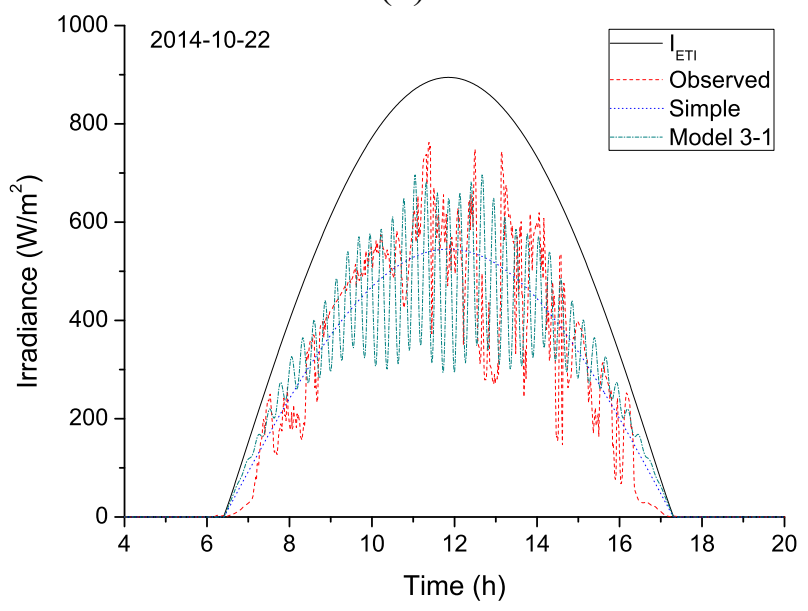

(c)

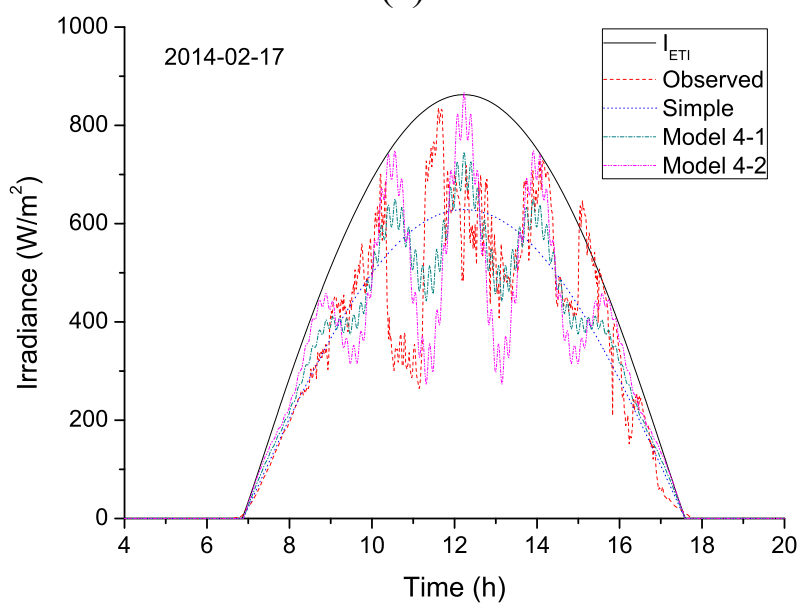

(e)

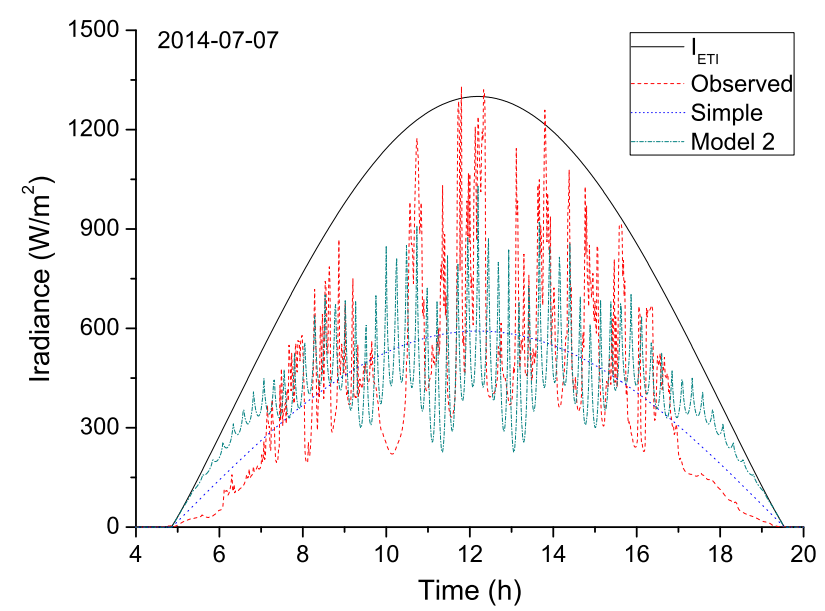

(b)

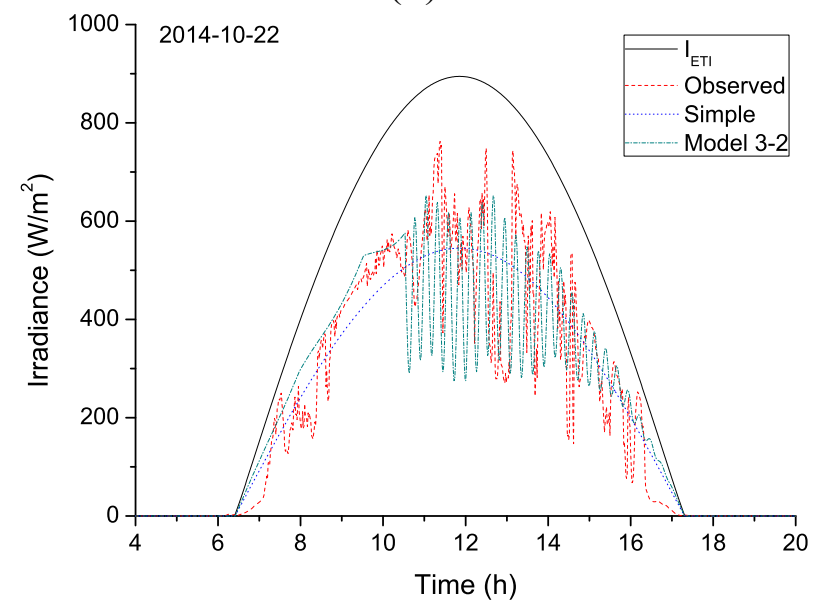

(d)

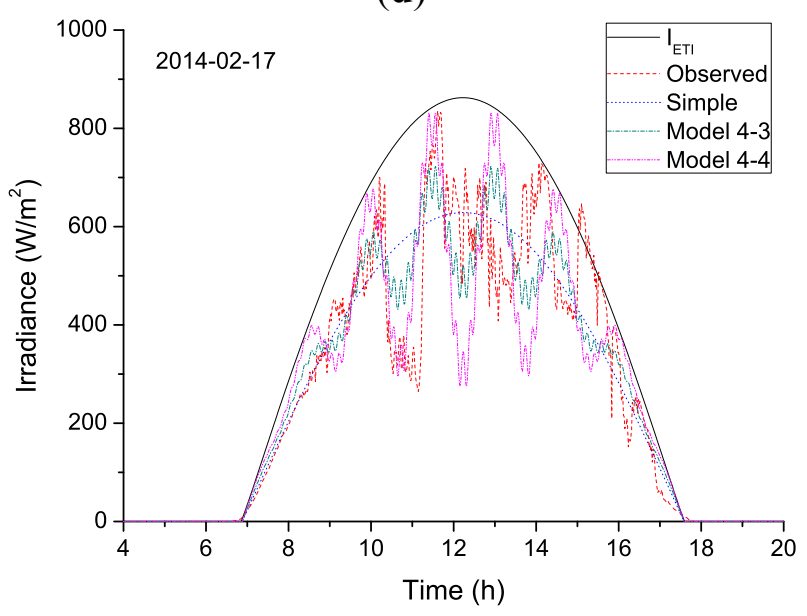

(f)

Figure 2. Solar radiation distributions for the actual weather data, the simple sky model and the proposed model as shown in Table 2. Data (a-d) observed in Anatolia-Rancho Cordova, California (latitude: $38.546^{\circ} \mathrm{N}$; longitude: $121.24^{\circ} \mathrm{W}$; elevation: $51 \mathrm{~m}$; PST) [20]. Data (e,f) observed in Golden, Colorado (latitude: $39.742^{\circ} \mathrm{N}$; longitude: $105.18^{\circ} \mathrm{W}$; elevation: $1828.8 \mathrm{~m}$; MST) [19]. 
Table 2. Solar radiation simulation variables and indices.

\begin{tabular}{|c|c|c|c|c|c|c|c|c|c|c|c|}
\hline \multirow{2}{*}{ Data } & \multirow{2}{*}{ Model } & \multicolumn{7}{|c|}{ Variables } & \multicolumn{3}{|c|}{ Indices } \\
\hline & & $M$ & $a_{1}$ & $b_{1}$ & $c_{1}$ & $a_{2}$ & $b_{2}$ & $c_{2}$ & $k_{D}$ & $\boldsymbol{P O P}_{D}$ & Class \\
\hline \multirow[t]{2}{*}{ Case 1} & Observed & & & & & & & & 0.67 & 0.98 & 1 \\
\hline & Simple & 0.67 & & & & & & & 0.67 & 0.99 & 1 \\
\hline \multirow[t]{3}{*}{ Case 2} & Observed & & & & & & & & 0.48 & 0.67 & 8 \\
\hline & Simple & 0.46 & & & & & & & 0.48 & 0.99 & 2 \\
\hline & Model & 0.81 & 0.2 & 8 & 3 & 0.6 & 60 & 3 & 0.48 & 0.67 & 8 \\
\hline \multirow[t]{4}{*}{ Case 3} & Observed & & & & & & & & 0.61 & 0.69 & 7 \\
\hline & Simple & 0.61 & & & & & & & 0.61 & 0.99 & 1 \\
\hline & Model 3-1 & 0.81 & 0.1 & 7 & 1 & 0.5 & 40 & 2 & 0.61 & 0.67 & 7 \\
\hline & Model 3-2* & 0.76 & 0.1 & 7 & 1 & 0.5 & 40 & 2 & 0.61 & 0.79 & 4 \\
\hline \multirow[t]{6}{*}{ Case 4} & Observed & & & & & & & & 0.72 & 0.78 & 4 \\
\hline & Simple & 0.73 & & & & & & & 0.72 & 0.99 & 1 \\
\hline & Model 4-1 & 0.86 & 0.3 & 6 & 2 & 0.1 & 64 & 2 & 0.72 & 0.90 & 1 \\
\hline & Model 4-2 & 1.01 & 0.6 & 6 & 2 & 0.1 & 64 & 2 & 0.72 & 0.84 & 4 \\
\hline & Model 4-3 & 0.86 & 0.3 & 7 & 2 & 0.1 & 64 & 2 & 0.72 & 0.89 & 4 \\
\hline & Model 4-4 & 1.01 & 0.6 & 7 & 2 & 0.1 & 64 & 2 & 0.72 & 0.81 & 4 \\
\hline
\end{tabular}

Note: * short-term fluctuation design at time 10:32 AM.

We investigate how different values of model parameters impact on the $k_{D}$ and $P O P_{D}$, and we evaluate the proposed model by comparing its results with weather conditions in the $k_{D}-P O P_{D}$ classes. Figure 3 shows the dependence of $k_{D}, P O P_{D}$ and $k_{D}-P O P_{D}$ classes on the parameters of the variable weather model given by Equation (4). The values of $k_{D}$ are strongly affected by the changes in the $M, a_{1}$ and $c_{1}$ values, while the values of $P O P_{D}$ are mainly affected by the changes in the $b_{1}$ parameter. Thus, the results presented in Figure 3 show that the proposed model can realize a wide range of weather changes for solar energy system simulations. Although the $k_{D}-P O P_{D}$ Classes 6, 7 and 9 do not appear in the figure, the solar radiation distributions in those classes can be easily obtained by simultaneously changing two or more variables in Equation (4).

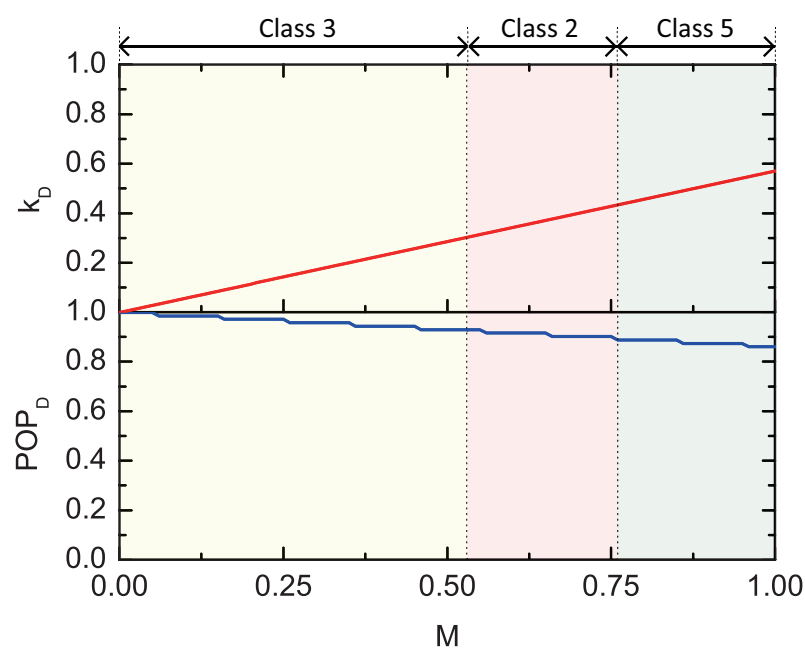

(a)

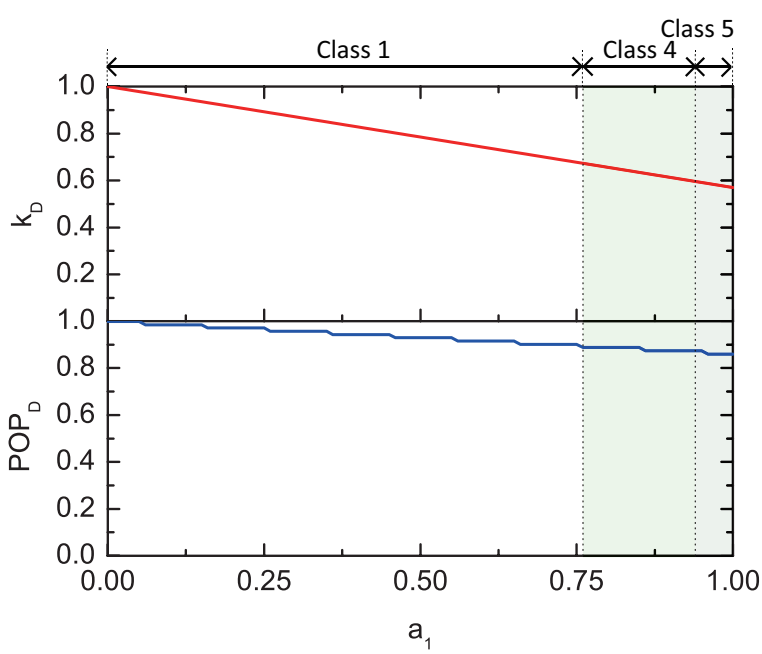

(b)

Figure 3. Cont. 


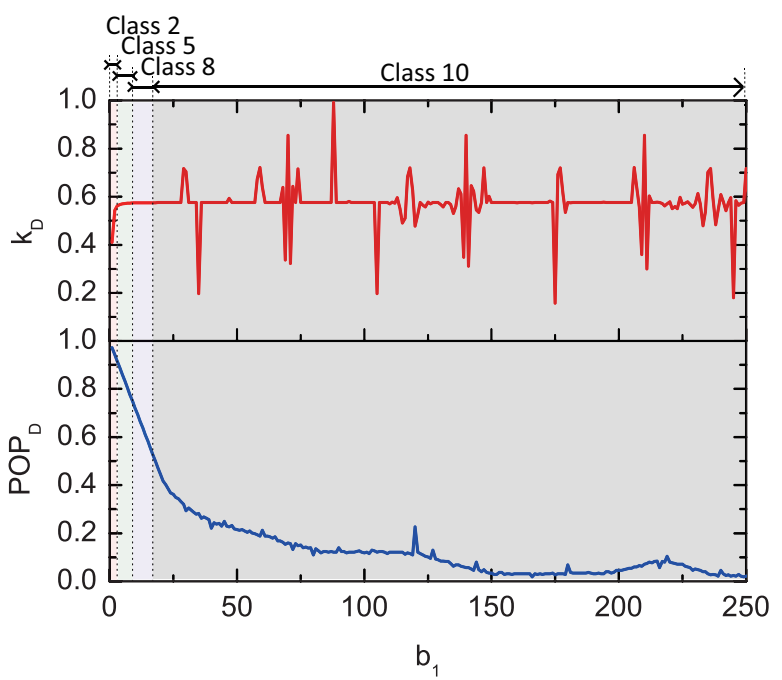

(c)

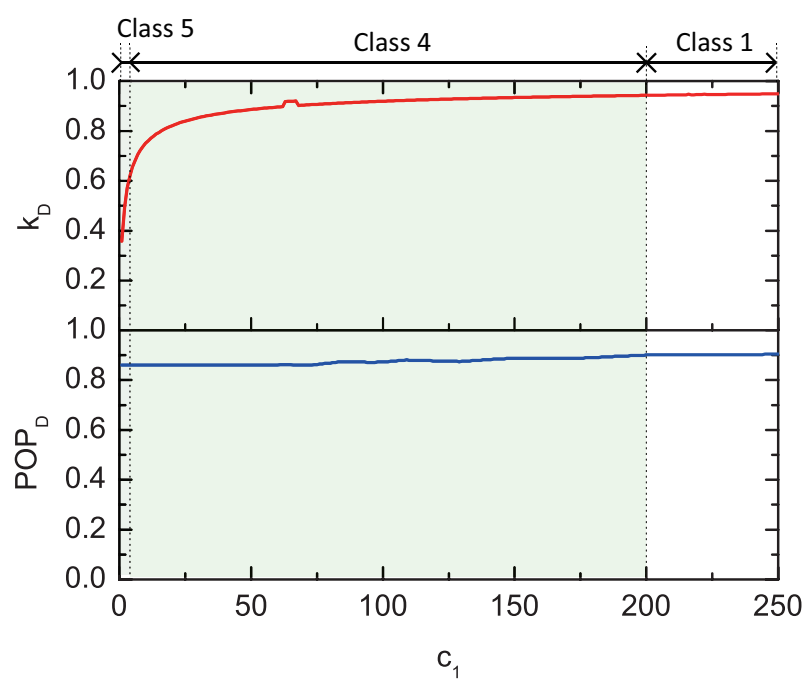

(d)

Figure 3. Effects of the changes in (a) $M$, (b) $a_{1}$, (c) $b_{1}$ and (d) $c_{1}$ on $k_{D}, P O P_{D}$ and the $k_{D}-P O P_{D}$ class for the base model with $M=1, a_{1}=0.5, a_{2}=0, b_{1}=5, b_{2}=0, c_{1}=3$ and $c_{2}=3$.

The weather simulation results show that the proposed model implements actual weather conditions to limited extent. In spite of these limitations, the proposed model could be applied to thermal system simulation, since the proposed model provides a tool for case studies beyond the intrinsic randomness of weather.

\section{Application: Dynamic Simulation of SORC}

In this chapter, a dynamic simulation of a solar organic Rankine cycle (SORC) system is conducted to figure out the differences between the simple model and the proposed model. The schematics of the SORC system is shown in Figure 4. The ORC system is a type of Rankine cycle system that uses organic refrigerant as the working fluid. The change of the solar irradiance influences the operating characteristics of the SORC system, such as solar collector efficiency, thermal efficiency and system on/off frequency.

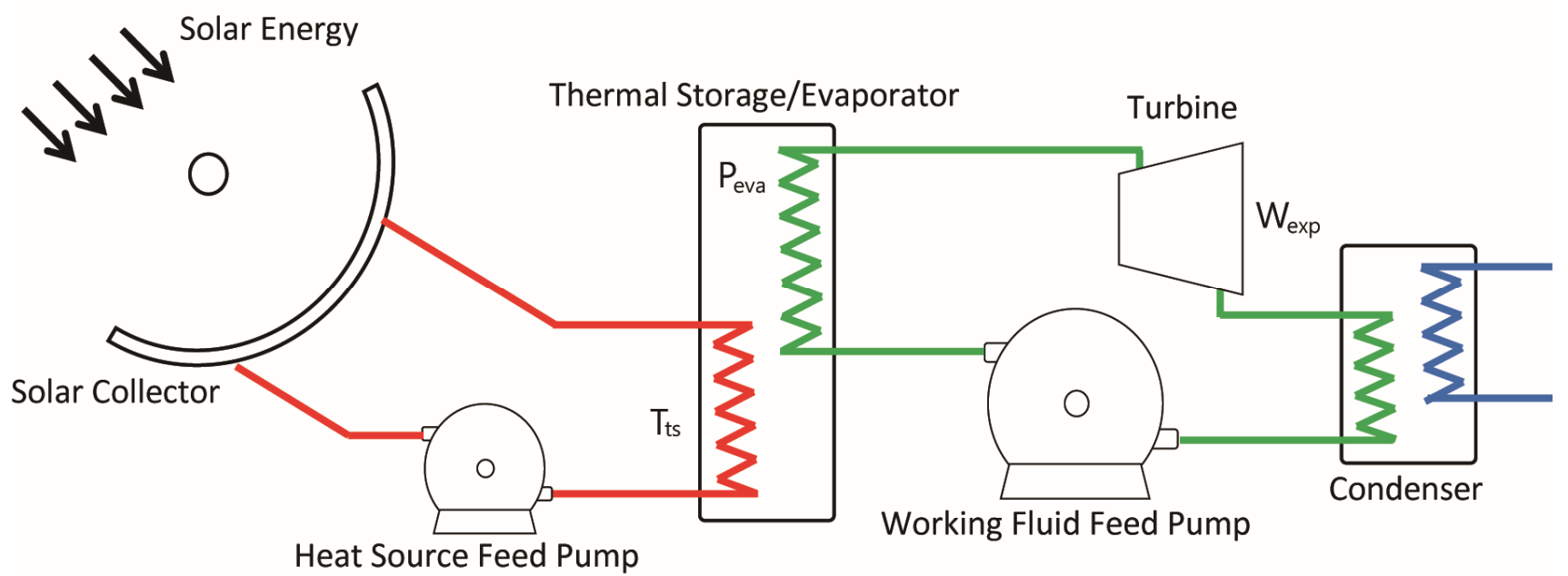

Figure 4. Schematic diagram of the solar organic Rankine cycle (SORC) model. 
A lumped SORC model designed by Twomey et al. for studying the relationship between the solar radiation pattern and the SORC response [21] was adopted in this simulation. In this study, thermal storage is adopted, and it stabilizes the system operation from weather variability. Such an effect is studied by Wang et al. [22]. The SORC model expressed is as energy balance between solar cycle, ORC and heat losses as follows:

$$
\frac{d U_{s t}}{d t}=\dot{Q}_{s o l}-\dot{Q}_{u h x}-\dot{Q}_{s t a n d}
$$

where $U_{s t}$ is the thermal storage internal energy, $\dot{Q}_{s o l}$ is the useful heat gain from solar collector, $\dot{Q}_{u h x}$ is the heat transfer to the ORC and $\dot{Q}_{s t a n d}$ is the standing heat losses (for a more detailed description of the modelling, see Twomey et al. [21]). Considering solar collector performances, the SORC system was designed to be activated at a storage temperature of $120{ }^{\circ} \mathrm{C}$ and deactivated at $85{ }^{\circ} \mathrm{C}$. Small-scale SORC system studies [22,23] show that R245fa (1,1,1,3,3-pentafluoropropane) refrigerant has good performance in low and medium heat source temperatures; therefore, R245fa refrigerant was selected as the working fluid. For stable and continuous system operation, the pressure ratio of three was applied (the adopted pressure ratio is relatively low compared to other small-scale SORC studies [22-25]). The remaining thermal energy in the thermal storage after sunset was used for additional power generation. The shaft power $\left(\dot{W}_{\text {exp }}\right)$ is calculated by the product of working fluid mass flow rate $\left(\dot{m}_{r}\right)$ and the enthalpy difference between expander inlet $\left(h_{\text {exp,in }}\right)$ and outlet $\left(h_{\text {exp,out }}\right)$ as follows:

$$
\dot{W}_{\text {exp }}=\dot{m}_{r}\left(h_{\text {exp in }}-h_{\text {exp }, \text { out }}\right)
$$

To figure out the weather variability effect on the SORC system, long-term large weather changing conditions with short-term small fluctuations in Case 4 of Table 2 are simulated. The amount of the total daily solar irradiance $k_{D}$ for all model distributions was identical to the value for the actual distribution. Thus, system operation characteristics and power output are changed by the difference of solar irradiance distributions.

Figure 5 shows the simulation results for the thermal storage temperature and the shaft power. The results of storage temperature obtained both using the actual weather data and as predicted by the proposed model exhibit large variations and local overshoots, while the storage temperature predicted by the simple sky model shows a smooth behavior with significantly less variation. Thus, the temperature and shaft power results show that the proposed model provides better system operation characterization, including operation mode changes, than the simple sky model. In all cases, the amount of daily total solar irradiances is the same; however, the operating characteristics are dramatically changed according to the shape of solar irradiance distributions. In Cases 4-2 and Case 4-4, the system is turned off around noon, while in Cases 4-1 and 4-3, the model is not turned off, as an illustration of simple cases. Table 3 lists the daily work total and its differences compared with the results of the observed data. In Case 4-4, weather variability does not match the observation when clouds appear, which results in different operating characteristics and the largest difference in daily work total. The case study results show that the proposed model does not perfectly express the actual observed weather condition and its system responses. However, it is possible to test a variety of weather conditions according to the study object. 


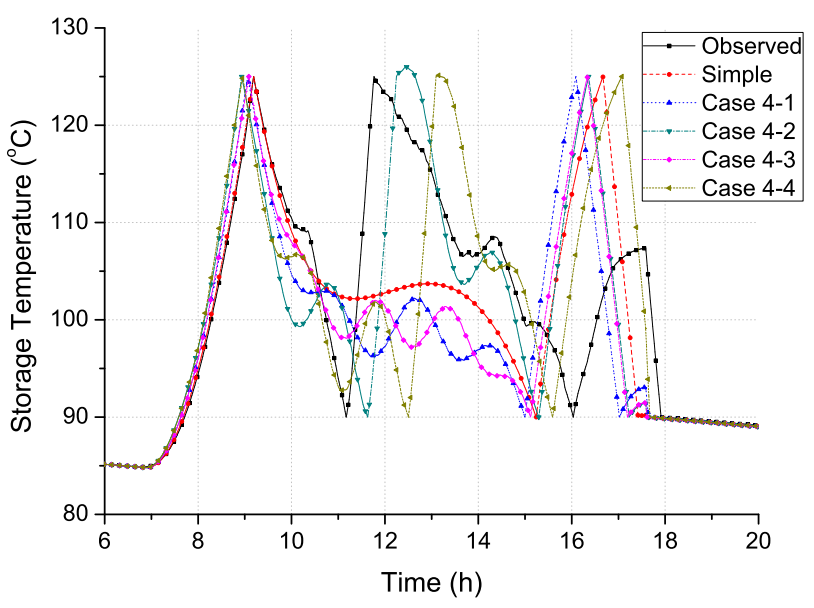

(a)

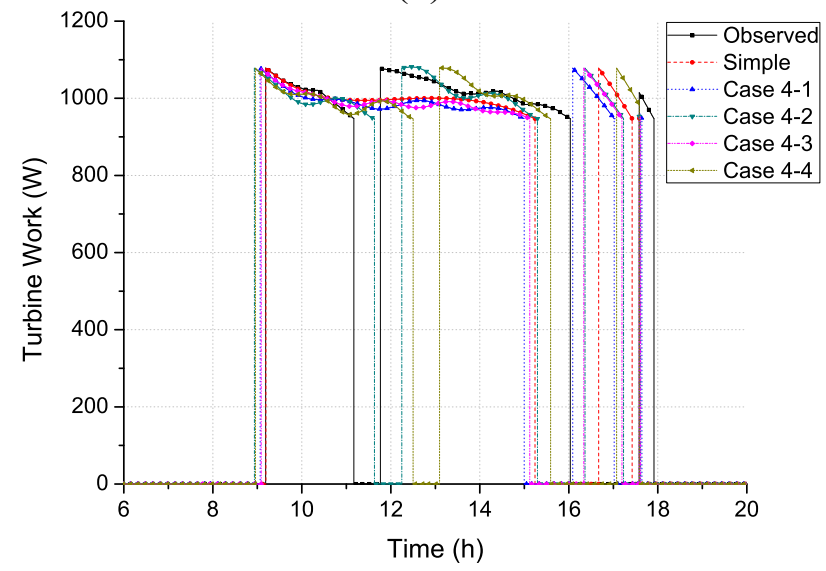

(b)

Figure 5. (a) Thermal storage temperature and (b) shaft power for the actual weather conditions, the simple sky model and the proposed model.

Table 3. Daily work total and its differences between the results of observed data and the others.

\begin{tabular}{ccccccc}
\hline Parameters & Observed & Simple & Case 4-1 & Case 4-2 & Case 4-3 & Case 4-4 \\
\hline Work Total $(\mathrm{kWh})$ & 6.68 & 6.81 & 6.88 & 6.71 & 6.89 & 6.89 \\
Difference $(\%)$ & 0.00 & 1.92 & 2.93 & 0.43 & 3.04 & 3.04 \\
\hline
\end{tabular}

\section{Conclusions}

A mathematical model of the hourly solar radiation for a cloudy sky was proposed and evaluated using the clearness index $k_{D}$ and the probability of persistence $P O P_{D}$. We found that the proposed model could realize all classes of the $k_{D}-P O P_{D}$ classification scheme suggested by Kang and Tam [18]. Thus, the proposed model can treat a wide range of weather changes in simulations of solar energy systems. As an illustration, the proposed model was applied to the dynamic simulation of a SORC. The simulation results showed that the proposed model could provide better system operation characteristics than the simple sky model for significant variations in weather conditions. The proposed model in this study is the first model of weather variability; we expect that further investigations will improve the model and enable more effective design and development of solar energy systems. 


\section{Acknowledgments}

This research was partially supported by the Basic Science Research Program of the National Research Foundation of Korea (NRF) funded by the Ministry of Education (NRF-2013R1A1A2012173); partially by the Energy Efficiency and Resources of the Korea Institute of Energy Technology Evaluation and Planning (KETEP) and granted financial resource from the Ministry of Trade, Industry \& Energy, Republic of Korea (No. 20112010100030); and partially by the National Research Foundation of Korea (NRF) in a grant funded by the Korea government (MSIP) through Global Core Research Center for Ships \& Offshore Plants (GCRC-SOP, No. 2011-0030013).

\section{Author Contributions}

All authors contributed to this work by collaboration. Taehong Sung is the first author in this manuscript. The whole project was supervised by Sang Youl Yoon and Kyung Chun Kim. All authors revised and approved for the publication.

\section{Conflicts of Interest}

The authors declare no conflict of interest.

\section{References}

1. Kalogirou, S.; Lloyd, S.; Ward, J.; Eleftheriou, P. Design and performance characteristics of a parabolic-trough solar-collector system. Appl. Energy 1994, 47, 341-354.

2. Tian, Y.; Zhao, C.-Y. A review of solar collectors and thermal energy storage in solar thermal applications. Appl. Energy 2013, 104, 538-553.

3. Iqbal, M. An Introduction to Solar Radiation; Elsevier: New York, NY, USA, 1983.

4. Atwater, M.A.; Ball, J.T. A surface solar radiation model for cloudy atmospheres. Mon. Weather Rev. 1981, 109, 878-888.

5. Iqbal, M. Correlation of average diffuse and beam radiation with hours of bright sunshine. Sol. Energy 1979, 23, 169-173.

6. Weiss, A.; Hays, C.J. Simulation of daily solar irradiance. Agric. For. Meteor. 2004, 123, 187-199.

7. Yang, K.; Huang, G.; Tamai, N. A hybrid model for estimating global solar radiation. Sol. Energy 2001, 70, 13-22.

8. Yang, K.; Koike, T. Estimating surface solar radiation from upper-air humidity. Sol. Energy 2002, 72, 177-186.

9. Angstrom, A. Solar and terrestrial radiation. Report to the international commission for solar research on actinometric investigations of solar and atmospheric radiation. Q. J. R. Meteorol. Soc. 1924, 50, $121-126$.

10. Prescott, J. Evaporation from a water surface in relation to solar radiation. Trans. R. Soc. S. Aust. 1940, 64, 114-118.

11. Ehnberg, J.S.; Bollen, M.H. Simulation of global solar radiation based on cloud observations. Sol. Energy 2005, 78, 157-162.

12. Supit, I.; van Kappel, R. A simple method to estimate global radiation. Sol. Energy 1998, 63, 147-160. 
13. Bechini, L.; Ducco, G.; Donatelli, M.; Stein, A. Modelling, interpolation and stochastic simulation in space and time of global solar radiation. Agric. Ecosyst. Environ. 2000, 81, 29-42.

14. Bristow, K.L.; Campbell, G.S. On the relationship between incoming solar radiation and daily maximum and minimum temperature. Agric. For. Meteorol. 1984, 31, 159-166.

15. Thornton, P.E.; Running, S.W. An improved algorithm for estimating incident daily solar radiation from measurements of temperature, humidity, and precipitation. Agric. For. Meteorol. 1999, 93, 211-228.

16. Freeman, J.; Hellgardt, K.; Markides, C.N. An assessment of solar-thermal collector designs for small-scale combined heating and power applications in the united kingdom. Heat. Transfer. Eng. 2015, $36,1332-1347$.

17. Reda, I.; Andreas, A. Solar position algorithm for solar radiation applications. Sol. Energy 2004, 76, 577-589.

18. Kang, B.O.; Tam, K.-S. A new characterization and classification method for daily sky conditions based on ground-based solar irradiance measurement data. Sol. Energy 2013, 94, 102-118.

19. Stoffel, T.; Andreas, A. Nrel Solar Radiation Research Laboratory (srrl): Baseline Measurement System (bms); Ggolden, Colorado (data); National Renewable Energy Laboratory (NREL): Golden, CO, USA, 1981.

20. Maxey, C.; Andreas, A. Sacramento Municipal Utility District (smud): Rotating Shadowband Radiometer (rsr); Anatolia-rancho Cordova, California (data); National Renewable Energy Laboratory (NREL): Golden, CO, USA, 2009.

21. Twomey, B.; Jacobs, P.; Gurgenci, H. Dynamic performance estimation of small-scale solar cogeneration with an organic rankine cycle using a scroll expander. Appl. Thermal Eng. 2013, 51, 1307-1316.

22. Wang, M.; Wang, J.; Zhao, Y.; Zhao, P.; Dai, Y. Thermodynamic analysis and optimization of a solar-driven regenerative organic rankine cycle (orc) based on flat-plate solar collectors. Appl. Thermal Eng. 2013, 50, 816-825.

23. Wang, M.; Wang, J.; Zhao, P.; Dai, Y. Multi-objective optimization of a combined cooling, heating and power system driven by solar energy. Energy Convers. Manag. 2015, 89, 289-297.

24. Villarini, M.; Bocci, E.; Moneti, M.; Di Carlo, A.; Micangeli, A. State of art of small scale solar powered orc systems: A review of the different typologies and technology perspectives. Energy Procedia 2014, 45, 257-267.

25. Freeman, J.; Hellgardt, K.; Markides, C.N. An assessment of solar-powered organic rankine cycle systems for combined heating and power in uk domestic applications. Appl. Energy 2015, 138, $605-620$.

(C) 2015 by the authors; licensee MDPI, Basel, Switzerland. This article is an open access article distributed under the terms and conditions of the Creative Commons Attribution license (http://creativecommons.org/licenses/by/4.0/). 